Voix et Images

voixetimages

\title{
Un jour on va revenir à la poésie. Entretien avec Gilles Hénault
}

\section{Paul Chamberland}

Volume 21, numéro 1 (61), automne 1995

Gilles Hénault

URI : https://id.erudit.org/iderudit/201211ar

DOI : https://doi.org/10.7202/201211ar

Aller au sommaire du numéro

Éditeur(s)

Université du Québec à Montréal

ISSN

0318-9201 (imprimé)

1705-933X (numérique)

Découvrir la revue

Citer ce document

Chamberland, P. (1995). Un jour on va revenir à la poésie. Entretien avec Gilles Hénault. Voix et Images, 21(1), 12-23. https://doi.org/10.7202/201211ar d'utilisation que vous pouvez consulter en ligne.

https://apropos.erudit.org/fr/usagers/politique-dutilisation/ 


\section{Un jour on va revenir à la poésie Entretien avec Gilles Hénault*}

\section{Paul Chamberland, Université du Québec à Montréal}

La poésie coule dans la plaine où s'abreuvent les peuples.

V. et I. : Beaucoup de tes poèmes font penser à des tableaux. J'avais noté dans le poème "Fusée": "Surgissent d'hybrides pétales, des cristaux de flammes, neigeant sur des soleils emplumés". J'avais trouvé que ça évoquait soit Bellefleur soit Riopelle, mais je dirais que c'est Bellefleur dans ce cas-ci.

G. H. : On y voit un Bellefleur.

V. et I. : Comment cet intérêt pour l'art est-il né?

G. H.: Depuis ma tendre enfance, j'ai toujours été fasciné par le côté "magique" de la peinture. Tout jeune, j'ai fait un peu de sculpture, mais c'était très mauvais (rires). Je m'en suis aperçu à temps (rires). En 1939, j'ai gagné un prix octroyé par le journal Le Canada pour mon travail de journaliste et je suis allé à l'Exposition universelle à New York en 1939. Je me souviens d'une toile de Rembrandt, le Saint-Pierre. J'avais dix-huit ans, dix-neuf ans... À vrai dire, c'était mon premier regard sur la peinture, çavait été une révélation.

V. et I. : Tu as commencé à écrire tôt?

G. H.: J'ai commencé à écrire à quatorze ou quinze ans, comme la plupart des jeunes qui font leurs premières armes.

V. et I.: Quand on commence à écrire, il y a toujours des déclencheurs, les émotions, le contexte... Mais aussi la rencontre d'un poète, ou de deux ou trois poètes... Pour toi, qui est-ce?

G. H. : Probablement Villon. Mais comme tu dis, il y a toujours plusieurs personnes qui sont là, il y a tout un cénacle...

- Participait à l'entretien Lise Demers, compagne du poète; ses interventions ont été d'un précieux apport. Le tutoiement a été expressément voulu par Gilles Hénault. La transcription a été effectuée par Andrée DeRome. 
V. et I.: André-G. Bourassa, dans son ouvrage Surréalisme et littérature québécoise, fait allusion à des auteurs comme Paul Váléry, par exemple...

G. H.: Oui, j'ai été marqué par des poèmes, comme La Jeune Parque, et plusieurs ouvrages... Eupalinos ou l'Architecte, mais beaucoup plus tard... Il y a eu aussi Apollinaire, Rimbaud, Verlaine, Supervielle...

L. D.: Quand tu étais jeune, il y a aussi Alexis Carrel qui t'a influencé. L'Invention de la roue, c'est un peu un hommage à Alexis Carrel...

G. H.: On peut dire ça, oui. L'Homme, cet inconnu, c'était vraiment une somme pour moi, une somme scientifique. Je trouvais ça intéressant.

V. et I. : Par ailleurs, et on peut voir la filiation, il y a Alain Grandbois...

G. H.: Ah oui! C'était avant, assez jeune. Les îles de la nuit... C'était pour moi une découverte.

V. et I.: Quand on pense au voyage, au titre d'abord, Voyage au pays de mémoire, mais aussi au fait qu'on "voyage " dans tes poèmes, on peut te voir comme le relais d'un poète comme Grandbois. Est-ce que la fascination du voyage pouvait jouer?

G. H.: Oui. D'ailleurs, le premier voyage que j'ai fait, c'est à bord d'un bateau, un paquebot qui transportait des chevaux. Riopelle aussi l'a fait.

L. D.: Gilles était palefrenier, il s'occupait des chevaux sur le bateau et c'est ce qui payait le voyage. Les chevaux étaient envoyés en France, c'était tout de suite après la guerre, pour servir dans les boucheries chevalines, parce que les Français avaient besoin de victuailles. (Pause.) Tu étais revenu d'Europe avec Paroles de Jacques Prévert, qui venait tout juste de sortir des presses...

G. H. : Prévert m'avait intéressé. Sa poésie le démarquait... son humour.

V. et I. : À ce moment-là, tu avais déjà commencé à lire Breton, qui avait élaboré la théorie du surréalisme, ou Éluard? Ont-ils été marquants?

G. H.: Oui, mais sur des plans différents, parce que je n'aimais pas beaucoup la théorie.

V. et I. : La poésie d'Éluard te touchait davantage que la poésie de Breton.

G. H. : Oui. J'appréciais la simplicité d'Éluard. C'est peut-être par le biais de Roland Giguère... Mais déjà circulait dans le milieu Aimé Césaire, Les Armes miraculeuses. C'est par New York que ça nous arrivait... Césaire situait les surréalistes dans un nouveau contexte. Poète du nouveau monde. Breton n'est pas un bon poète; Césaire est un très bon poète. Il a été pour moi un déclencheur, parce que je reconnaissais tout à coup un autre, mais un autre qui n'était pas français, et qui était quand même parent.

V. et I. : Dès le départ, Césaire a eu une place privilégiée ici... 
G. H. : Le premier qui me l'a fait connaître, c'est Mousseau, assez curieusement. Par l'intermédiaire de Louise Renaud, qui vivait à New York à ce moment-là.

V. et I. : Tu as dû aussi prendre connaissance du Cabier d'un retour au pays natal?

G. H.: Oui, ç'a été pour moi très important. Le Cabier... était très significatif de ce que pourrait être la poésie.

V. et I.: Il y a une présence de Rimbaud dans ta poésie. Je pense aux Illuminations. On retrouve le pictural...

G. H.: Oui. Mais aussi dans le poème "Voyelles": pour moi, ce poème équivaut à un petit tableau.

V. et I. : En 1946, tu fondes les Cabiers de la file indienne avec Éloi de Grandmont et tu y fais paraître Théâtre en plein air avec des illustrations de Daudelin. Comment, à l'époque où tu écrivais ces poèmes, jouait la vision du surréalisme... l'automatisme, le rêve?

G. H. : Pour moi c'était assez intégré. Il y a des bonshommes qui m'ont marqué plus que d'autres, Robert Desnos particulièrement. Ses expériences et aussi sa pensée, qui m'était beaucoup plus familière. Et il était très impliqué dans les questions sociales. C'était un communiste.

V. et I. : Est-ce que Duchämp a compté également?

G. H.: Oui, mais plus tard. Quoique, évidemment, il était présent à New York. J'appréciais son humour et ce qui en a résulté.

V. et I.: Les surréalistes sont en Amérique pendant la guerre. Certains prennent contact avec les "surréalistes" d'ici, surtout les peintres: Pellan, Riopelle...

G. H. : Plutôt Leduc... à cause de Louise Renaud, qui a été le lien. Et il y a eu une sorte de jonction. Mais chacun s'est démarqué dans sa façon d'être.

V. et I. : Tu t'es retrouvé très rapidement dans ce milieu des automatistes, notamment avec Daudelin.

L. D.: Connaissais-tu Borduas à l'époque de l'exposition des "gouaches de 42 "?

G. H. : Non, je l'ai connu quelques mois plus tard, donc en 1942 ou 1943. Quand j'ai vu pour la première fois les gouaches ou les toiles de Borduas, j'ai été désarçonné. Riopelle m'était plus familier.

V. et I. : Est-ce que ça a été durable?

G. H. : Oui. Il a développé son processus et c'est resté Riopelle.

V. et I. : C'était l'époque, en 1946, où il y a eu en France "rupture inaugurale" entre la position de Breton et celle des autres: le surréalisme doit 
rester libre de tout engagement, au Parti communiste notamment. Le même débat va faire rage ici. Borduas a toujours été catégorique là-dessus. Toi, tu t'inscris au Parti communiste et par ailleurs tu n'as pas signé le Refus global... Comment les choses se sont-elles passées avec Borduas et les autres?

G. H. : Justement, pour Borduas, il n'y avait pas de compromis.

V. et I.: Tu as quand même défendu la position des automatistes et le Refus global, dès sa parution, face aux réactions de la "bêtise ambiante " et face aux communistes. Ta position était donc très nuancée...

G. H. : Je me prononçais en faveur du surréalisme mais aussi, c'était une espèce de compromis, assez curieusement... J'ai, par exemple, conservé comme amie Marcelle Ferron. Jacques Ferron était aussi au Parti. Il fallait que je me maintienne à la fois dans le Parti et hors du Parti, car je trouvais que c'était devenu moche.

L. D.: Ce que j'ai cru comprendre, c'est qu'il était très difficile de proposer devant Borduas une opinion différente de la sienne. Fernand Leduc a essayé et les portes ont claqué... C'était un peu la même chose avec Riopelle, quand il proposait au groupe de Montréal d'exposer avec les surréalistes: tout de suite Borduas, comme un chef d'équipe, si on veut, a opposé son véto et si les autres allaient à l'encontre, ils essuyaient ses foudres.

G. H. : Oui, mais c'est plus complexe encore. Il y avait les surréalistes français. Desnos avait été favorable à l'engagement révolutionnaire, au Parti ; une-bonne partie de ces gens-là l'étaient toujours après la guerre:

V. et I. : Il y a eu chez toi une importante action syndicale. Dans le premier numéro de Chroniques, lors d'un entretien avec Philippe Haeck, Jean-Marc Piotte et Patrick Straram, on parlait de ton action avec les mineurs de Sudbury et tu disais qu'au Québec tu n'avais pas vraiment eu ta place, qu'il n'y avait pas grand-place pour les syndicats de gauche. C'est pourquoi tu t'étais retrouvé dans le syndicat des mines, le Mines Mills and Smelter Workers of America, lui-même affilié à une centrale américaine. Tu as été combien de temps à Sudbury?

G. H. : Cinq ans, à partir de 1952.

V. et I.: Qu'est-ce que tu retiens de toute cette action avec les mineurs? Dans tes poèmes, ça compte beaucoup; ç'a dû être déterminant dans ton engagement...

G.' H.: Oui, sauf qu'en même temps, j'étais là et pas là. Je comprenais les mineurs mais je me disais que c'était autre chose que je pouvais faire le mieux.

V. et I. : Un peu en exil, peut-être?

L. D.: Il a été effectivement en exil. Si Gilles et sa famille ne sónt pas morts de faim à l'époque, c'est parce que Bernard Jasmin leur donnait à 
manger. Avant qu'il aille à Sudbury, il travaillait un peu incognito ici, avec Léa Robach, qui a fait des émissions sur les syndicats d'électricité, et aussi pour le syndicat de la " sacoche" (rires). C'étaient des syndicats de gauche, et ç'a fini par être dénoncé dans les journaux. Gilles a perdu tous ses jobs. Il a travaillé aussi à l'époque avec Madeleine Parent, lors de la grève du textile, déclenchée par le syndicat Dominion Textile. Lorsque les syndicats ont été lessivés du Québec, plus personne n'osait lui donner le moindre job. C'est pour ça qu'il a été obligé de s'exiler à Sudbury.

V. et I. : Quels étaient tes rapports avec Gauvreau, aussi bien sur le plan personnel que par rapport à sa production? Vous n'avez pas eu beaucoup de contacts?

L. D.: Oui, il a éu des contacts avec Gauvreau. Il a même répété dans une de ses pièces. Et quand Gauvreau a été interné à un moment donné, il était là... Avec Marcelle Ferron, il a essayé de le faire sortir.

V. et I. : Ça jouait à deux niveaux... Si j'ai bien compris, la divergence se situait sur le plan esthétique?

G. H. : Oui, mais Gauvreau était "ailleurs", quand même...

V. et I. : Comment en es-tu arrivé à ce que Dumouchel illustre Totems et plus tard, Marcelle Ferron Voyage au pays de mémoire? Ça s'est fait naturellement?

G. H.: Oui. Il faut dire que Dumouchel se foutait pas mal de mes positions politiques. Lui, il faisait de l'art et ça lui suffisait. Je pense que c'est Giguère d'ailleurs qui m'avait suggéré Dumouchel... Pour Giguère, mes positions politiques étaient difficiles à prendre, c'est-à-dire qu'il ne comprenait pas.

L. D. : Il aimait ta poésie, c'est ce qui te sauvait toujours...

V. et I. : Il y a une parenté entre Giguère et toi, une différence totale de style mais une sorte de proximité. Comme si ce que vous êtes allés chercher dans le surréalisme était assez proche.

G. H. : Oui, c'est vrai.

V. et I. : Lorsque Paul-Marie Lapointe, très jeune, a publié Le Vierge incendié, qu'est-ce que ce texte a représenté pour toi?

G. H. : Je n'ai pas connu le texte tout de suite. Évidemment j’ai reconnu ce travail comme étant majeur, mais après coup.

V. et I.: Si je regarde tes poèmes, ça ne date pas. Commençons par le début, L'Invention de la roue, par exemple. Quel âge avais-tu quand tu l'as écrit?

G. H. : Probablement... dix-huit, dix-neuf ans. Ça date de 1939-1940.

V. et I. : Ce qui me frappe dans cette ouvre, c'est que toutes les grandes figures qui apparaîtront dans ta poésie par la suite, on les sent déjà. "Et 
bâtir de mes mains cette Cité future / Où courbe, s'inscrirait la marque du compas".

G. H.: Oui, je pense que c'est assez évident, ça va vraiment déjà vers le progrès.

V. et I. : Si je pense à "Fusée", dans Voyage..., on sent une foi optimiste dans la science et la technique. Tu parlais plus tôt d'Alexis Carrel...

G. H.: Carrel m'a inspiré parce qu'il me mettait en disponibilité: je trouvais que c'était vraiment ça l'avenir. Mais j'ai pas mal changé de cap depuis ce temps.

V. et I.: On imagine aussi que tu t'es plongé beaucoup dans les œuvres socialistes, peut-être Marx ou Lénine...

G. H.: Je n'ai pas beaucoup lu Marx ou Lénine: je trouvais ça fascinant quand même, mais ça ne s'inscrivait pas tout à fait dans mon schème de pensée.

V. et I. : Dans un passage du "Chant deuxième", tu parles du "désir infini d'un infini voyage " qui est à la fois conquête: "La lune! nous l'aurons, à nous, pauvres marins / Son occulte clarté... Comme la lune sera effectivement atteinte un peu plus tard, tu l'entrevoyais, cette conquête de l'espace?

G. H. : Pas vraiment. Pour moi, c'était quelque chose toujours à venir. Je ne pensais pas que ça pourrait venir si tôt.

V. et I. : Ces poèmes sont souvent écrits au futur... On y sent une force, une véhémence même.

G. H. : Je ne sais pas si c'est très réussi au point de vue formel; ça n'a pas autant de pouvoir que j'aurais voulu en avoir.

V. et I. : Avec Allégories, en 1943, et tout ce qui va suivre, tu abandonnes l'alexandrin pour passer ou au vers libre ou encore à la prose. Comment s'est fait ce passage?

G. H.: Je pense que j'avais l'impression de ne pas avoir épuisé la possibilité qui était là, que j'aurais pu faire plus. Pour moi, ça avait une résonance plus importante que ce que je pouvais faire à ce moment-là.

V. et I.: Ce qui n'avait plus de résonance t'a forcé à abandonner les vieilles règles?

G. H. : Oui.

V. et I.: On sent tout de suite, dans Allégories, que ta prose poétique s'affirme comme ce qu'elle sera par la suite. Elle est pleine déjà : ta respiration; ton souffle, la scansion...

G. H.: Je me demande s'il n'y aurait pas là une influence de Saint-John Perse auquel j'avais été initié par Jean-Aubert Loranger qui travaillait à $L a$ 
Presse à ce moment-là. Loranger m'avait dit qu'il l'aimait beaucoup. Ç'a été une influence déterminante. Plus tard, à l'époque de Signaux... ça continuait à me hanter.

V. et I. : Est-ce que, dans les années quarante, au moment où tu écris, les images se bousculent? Il y a de l'onirisme... Quand tu écris les poèmes de Théâtre en plein air, en 1946, c'est très évident. Est-ce qu'il y a des moments intensifs d'écriture?

G. H. : Ça n'a jamais été tellement intensif. C'est comme si c'était donné, ça se révèle là et c'est tout. Je ne refais pas, c'est toujours un premier jet.

V. et I. : C'est proche de la découverte surréaliste?

G. H. : Dans ce sens-là, oui.

V. et I.: Giguère et toi, comme vous êtes proches à l'époque, vous vous lisez vos poèmes?

G. H. : Bien sûr, il publie lui aussi et je vois ce qu'il fait. Mais c'est quand même très différent.

V. et I.: Ce qui semble en résonance, c'est cette ampleur qu'on retrouve chez Giguère, ce jaillissement de l'image.

G. H. : Lui, je pense, il a fait un seul vers qui soit, un peu, contestataire.

V. et I. : "La main du bourreau va finir par pourrir."

G. H. : C'est ça... mais lentement (rires).

V. et I.: Théâtre en plein air, en 1946, est illustré par Chàrles Daudelin. Entre les illustrations et tes poèmes, il y a un lien étroit. C'est plutôt une amitié qui est à la base de cette collaboration?

G. H.: C'est plutôt une amitié. Je cheminais encore. Probablement que pour lui, ce n'était pas un cheminement très important. Lui, il a continué à faire de la sculpture sans se référer à différents styles.

V. et I. : Il ne partageait pas ta vision d'avenir, à la fois onirique et révolutionnaire?

G. H.: La vision onirique, oui.

V. et I. : Dans Théâtre en plein air, cette fois, on a du vers libre. Mais on sent cette même ampleur de la scansion que dans la prose. Dans ce recueil, il y a aussi quelque chose qui apparaît davantage: la poésie amoureuse, érotique. Ça se passe comme avant? C'est donné, ça vient dans un premier jet?

G. H. : Peut-être pas, c'est peut-être plus organisé.

V. et I.: J'arrive à Totems, en 1953. Faisons un saut à la toute fin du recueil, au très beau poème "Je te salue": "Peaux-rouges / Peuplades disparues / dans la conflagration de l'eau-de-feu [...] Vous nous avez légué 
vos espoirs totémiques `. Ce qui est particulier, c'est qu'il n'y a rien de passéiste; c'est plutôt comme si tu allais aux racines...

G. H. : J'ai peut-être du "sauvage", je ne sais pas.

V. et I. : As-tu ce sentiment quand tu écris ces poèmes-là?

G. H.: Oui. Probablement. Très jeune, j'étais allé visiter l'Abitibi. J'y avais des tantes qui étaient religieuses et elles étaient toutes les deux gardemalades dans un hôpital. J'avais conçu à ce moment-là des façons de récupérer les histoires des Indiens. Je me souviens avoir écrit à ma tante pour lui demander des choses là-dessus. Ça vient de très loin.

V. et I. : As-tu toi-même rencontré des Indiens?

G. H. : Oui. Mais ça ne m'a rien donné par manque de temps.

V. et I. : Le paysage de l'Abitibi t'a-t-il marqué?

G. H.: Je ne pense pas, c'était plutôt les individus qui m'attiraient. Il me semble qu'on les appelait des "Têtes de boules".

V. et I. : Et ça été suffisant pour faire jaillir tout ce monde, au niveau de la poésie?

G. H. : Je ne sais pas. Peut-être que ma mère était indienne, ou métisse. En tout cas, c'était une tentative qui a peut-être été suffisante pour déclencher la suite.

V. et I. : Je passe à un type de poèmes tout à fait différent, "Chanson des mégots ", qui est un poème d'amour, mais humoristique. Qu'est-ce qui a pu donner naissance à ce poème?

G. H. : Probablement que mes femmes ont toujours fumé, et moi aussi je fumais à une époque. Mais c'était une pochade.

V. et I. : Un autre poème, un très beau poème, prend une résonance particulière si on le situe dans l'époque, c'est "Feu sur la bête-angoisse". Dans ce poème, est-ce qu'il s'agit de ce que tu vis personnellement ou s'il $\mathrm{y}$ a aussi un sentiment collectif?

G. H. : Je pense que c'est plutôt ce que je vis personnellement, parce que je fais vraiment allusion à mon fils, à autre chose que je vis à ce momentlà.

V. et I.: Voyage au pays de mémoire, cette fois c'est Marcelle Ferron qui va l'illustrer. Comment la collaboration se fait: en parallèle ou elle travaille d'après tes poèmes?

G. H. : Ce sont des disques, des tableaux, Folkways; on en a retenu deux pour le recueil. Il y a une correspondance mystérieuse, je ne sais pas laquelle, mais ça existe. Ce qu'elle fait, ça correspond.

V. et I.: Dans "Bestiaire", il y a quelque chose de nouveau. Je pense à ceci : "La poésie devrait [...] pétrir les choses, faire entendre au-dessus des 
cacophonies religieuses, philosophiques, morales et politiques le cri nu de l'homme qui affirme son existence singulière et grégaire. "Est-ce que, vis-à-vis ces discours, tu ressens une sorte d'impatience, de répugnance, à ce moment-là?

G. H.: Est-ce qu'il n'y aurait pas là une correspondance avec le Refus global?

V. et I. : Parlons maintenant de ces années où tu as été directeur du Musée d'art contemporain, de 1966 à 1971. As-tu réussi durant ces années à faire ce que tu avais le goût de faire?

G. H.: Oui, j'ai fait quelques expositions rétrospectives: Mousseau par exemple, Leduc, et d'autre part, j'ai eu des expositions assez importantes pour ce qui est de la démarche même, de la peinture. Je suis parti à cause de la maladie.

V. et I. : Tu as dû travailler à constituer la collection permanente, ce que Guy Robert avait commencé.

G. H. : Ce n'est peut-être pas la collection permanente qui a été le volet le plus important, ce sont davantage les rétrospectives. J'ai fait venir plusieurs œuvres d'ailleurs, d'un peu partout, Zao Wou-Ki notamment. Il y a eu plusieurs autres expositions, celle de l'art cinétique par exemple.

V. et I. : Cette passion pour l'art a-t-elle eu l'effet de réduire ta propre production? As-tu moins écrit durant cette période?

G. H.: J'ai certainement moins écrit parce que ça me prenàit tout mon temps ou presque.

V. et I. : Au-delà de l'organisation des événements et des tâches administratives, il fallait aussi favoriser des manifestations d'art, ça demandait de la vision.

G. H.: Oui, et j'étais très préoccupé par ça comme c'était le début du Musée, ou presque. C'était important que ce soit bien pensé.

V. et I. : As-tu trouvé que le milieu reconnaissait bien ta "mission"?

G. H. : D'une façon générale, j'ai eu une collaboration importante.

L. D. : Il faut dire que ce sont les artistes qui ont fait nommer Gilles. Au départ, il avait l'appui de toute la colonie artistique, et c'est ce qu'il avait demandé, il avait vérifié ça, sinon, il n'aurait pas pris le poste.

V. et I. : L'époque de ton directorat a aussi été celle de l'opération "Déclic". Comment as-tu vécu ça?

G. H. : J'ai vécu ça très cool, comme on dit. Je leur ai dit:' vous voulez cette opération, vous l'avez. C'était très perturbé.

V. et I.: Il y avait toute une turbulence et de nouveaux appétits du côté de l'art. C'étaient des jeunes et certains sont devenus des artistes majeurs, 
je pense à Serge Lemoyne notamment. On peut voir ça comme une réactivation de l'esprit du Refus global, j'imagine que tu étais quand même sensible à ça?

G. H. : Oui mais c'était un peu du chiqué, on ne refait pas ces choses-là.

L. D.: C'étaient eux qui avaient ressorti le Refus global et qui avaient occupé l'École des Beaux-arts. Ils avaient demandé, Serge Lemoyne avait demandé que tu viennes et tu t'étais rendu. Le ministre ne voulait pas parce qu'il disait que tu étais en conflit d'intérêts. Tu as quand même désobéi à ton ministre, Gilles (rires).

G. H. : Celui qui a succédé à Laporte, Cloutier, le "bon docteur "...

L. D.: Sous Jean-Noël Tremblay, il y a eu aussi toute l'histoire de la murale de Jordi Bonnet et de la phrase de Péloquin: "Vous êtes pas tannés de mourir, bande de caves ". Tremblay était de ceux qui voulaient faire ôter ça, il était ministre des Affaires culturelles à ce moment-là, et toi tu t'y étais opposé.

V. et I. : Et-ce que tu a dû soutenir une bataille assez forte?

G. H. : Oui. Jean-Noël était outré et voulait absolument que ça saute.

L. D. : Tremblay avait refusé l'achat d'une pièce de Lemoyne, qui doit être regardée au black light, ce n'était pas de l'art pour lui. Les fonds publics ne devaient pas aller à ce genre d'œuvres.

G. H. : Il faut dire qu'à certains moments il mettait son holà, parce qu'il en avait la possibilité. C'est bête parce que ce n'était pas le directeur du musée qui avait le droit d'exiger certaines choses, c'était le ministre. C'est complètement aberrant, parce qu'on avait un comité, qui avait recommandé l'achat de cette pièce.

V. et I.: Après Sémaphore, en 1962, on fait un saut jusqu'en 1984 , avec $\dot{A}$ l'inconnue nue. Est-ce que ça veut dire que tu as peu écrit dans l'intervalle?

L. D. : À l'inconnue nue était terminé en 1978.

V. et I. : Qu'est-ce qui t'a ramené à l'écriture?

G. H.: Toujours mes amis peintres. Bellefleur trouvait important de faire quelque chose avec ce texte.

V. et I.: Et s'il n'y avait pas eu l'impulsion de Bellefleur, tu n'aurais pas envisagé de les publier, ces poèmes?

G. H.: Je ne pense pas. Je n'en voyais pas la nécessité.

V. et I. : La collaboration de Bellefleur est quand même considérable, une dizaine de planches. Quand tu les a vues, tu as dit oui, ça devenait pour toi plus évident que ça pouvait être publié? 
L. D. : L'œuvre de Bellefleur accentue le côté érotique du texte, alors que pour toi, ce n'était pas nécesšairement un texte érotique. Il y a tout l'autre aspect du dialogue, d'égal à égal, que tu installes avec les autres poètes.

V. et I. : C'est un fait, on retrouve Rimbaud, Apollinaire, Nelligan, Mallarmé, Paul-Marie Lapointe... Est-ce que les phrases de ces poètes avaient beaucoup d'importance pour toi au moment où tu te mettais à écrire. Souvent elles sont au début des poèmes.

G. H.: Oui, parce que c'était en quelque sorte une consécration de ces poèmes.

V. et I. : Tu me disais des poèmes antérieurs que tu les écrivais tout d'un coup. C'a été la même chose pour ceux-là?

G. H.: Non, c'était beaucoup plus travaillé; parce que je partais de toutes ces phrases, et il était important pour moi d'aller chercher ce que je ressentais par rapport à elles.

V. et I. : Il y a de la jubilation dans ces poèmes-là. Il y a même un poème,

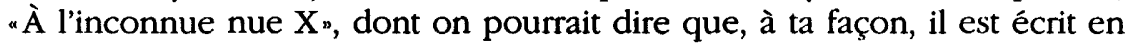
exploréen? C'est venu soudainement? Il n'y a pas de passage de Gauvreau cité, mais la manière Gauvreau qui est interpellée et reprise.

G. H. : Absolument.

L. D.: On pose souvent la question: "Quels poètes vous avez lus? Quels livres avez-vous préférés? " En fait, c'est un peu cette réponse-là qu'il donne. Au lieu de dire: "Il y a tel livre de telle personne, à telle époque....

V. et I. : Carroll ou Joyce...

G. H. : Oui, parce que c'est aussi un hommage à Joyce et à Carroll. J'avais la possibilité de lire le texte original. Joyce était pour moi très important.

V. et I.: Quand on arrive à à l'écoute de l'écoumène, il est intéressant de voir qu'à cause du temps, de l'époque, on n'est plus dans l'optimisme évolutionniste, disons, qui marquait tes premiers poèmes, mais plutôt face aux situations d'urgence de la planète. Qu'est-ce qui a déclenché un poème comme "Questions pour survivre"?

G. H.: Il est probablement né de toute cette façon de concevoir la civilisation.

V. et I.: “Écoumène ", le terme n'est pas très connu, mais il est néanmoins très juste.

G. H. : C'est le mot juste, mais en même temps, il fait le jeu de mots. " ̀̀ l'écoute de l'écoumènen, c'est vraiment la chose. Peut-être que j'aurais dû prendre un autre titre...

L. D.: Ça t'inquiète que les gens ne sachent pas ce que veut dire "écoumène , et qu'ils soient obligés de chercher dans le dictionnaire? 
G. H. : Peut-être, dans un sens, mais par contre je me dis que ça va devenir un mot connu. C'est d'ailleurs un mot très important, en géographie par exemple. J'ai vu des gens qui se sont butés sur le mot, qui ne savaient pas...

V. et I. : Dans ce qui t'a amené à faire ces exercices, comme tu les appelles, est-ce que la peinture de Zao Wou-Ki a joué un rôle?

G. H. : Non, pas vraiment.

V. et I. : Je pense à l'art chinois en général.

G. H.: La poésie chinoise m'avait impressionné et il y a aussi Roland Giguère qui avait un ami chinois et qui a fait un poème sur Tim Lum Lau.

V. et I.: Qu'il s'agisse de la poésie ou de l'art chinois, tu es fasciné par cela depuis longtemps?

G. H.: Oui, et je suis allé en Chine en 1970. J'y suis resté à peu près un mois. J'ai déjà commencé à apprendre le chinois. Mais finalement, contrairement aux enfants, qui apprennent ça en deux ou trois ans, nous on n'y arrive pas.

V. et I. : Présentement, crois-tu encore à la force de la poésie et de l'art dans notre société?

G. H.: Oui, je pense que c'est le seul recours. Peut-être qu'il y a aussi une façon différente de voir la poésie... On montrait il y a quelque temps que les Russes - toujours les Russes, malgré tout (rires) - ont découvert la possibilité, avec des miroirs, de faire un nouveau soleil. Ce n'est pas tout à fait ça... Un miroir assez vaste pour refléter le soleil dans les régions au nord. J'ai trouvé ça extraordinaire, parce que c'est une possibilité de remettre - je ne dirais pas le soleil à sa place (rires) - mais c'est une possibilité de voir le soleil autrement.

V. et I. : Mais aujourd'hui la réception de la poésie est très restreinte.

G. H. : Je pense qu'un jour on va revenir à la poésie.

V. et I. : Tu as confiance?

G. H.: Oui, parce qu'il y a beaucoup de phénomènes qui sont du mode poétique et qui sont là... Il faudra aller voir dans le dictionnaire, mais tant pis! 